\title{
Speculating on the Future of Graphic Design in the Age of Intelligent Machines
}

\begin{abstract}
Machine learning is set to have a profound impact on the graphic design industry in the near future. Despite its proximity, graphic design education and practice are largely sidelined from participating in the highly scientized spheres of computational aesthetics and applied image processing. Within this context, designer Sekyeong Kwon sought to make visible some of the cultural and practical implications of Al-powered design, from a graphic design perspective. The resulting practiceled project, Michael Barnes, falls within the subfield of adversarial design, and seeks to provoke contestation and debate around automation in design. The following short paper briefly sketches out the current graphic design landscape in relation to emerging technologies; outlines Michael Barnes; and explores a number of issues raised by the project including questions around (inter alia) aesthetics, authorship, and representation.
\end{abstract}

\section{Keywords}

Graphic Design, Automation, Adversarial Design, Practice-Led, Authorship

\section{Graphic Design and Automation}

The authorial hand of the graphic designer has always been indissolubly linked to the tools and technologies available to them. Since the mid-twentieth century, this causal relationship has been increasingly influenced by developments in human-computer interaction $(\mathrm{HCl})$, and more specifically, 'graphical user interfaces' [1]. MIT's Computer-Aided Design Project (1959-1967), for example, which spearheaded much of the early work into these systems, sought to create an interface which would "couple a man and a machine into a problem-solving team for fresh design problems" where each would perform "better than ... man or machine alone" [2].

More recently, however, and with developments in machine learning and deep learning in relation to computational aesthetics and aesthetic computing (see, for example, Google's DeepDream, MIT's Nightmare Machine) the view of computer-aided design software as an aid, is being superseded by its potential to make autonomous or semi-autonomous creative decisions [34]. Applied instances of embedded artificial intelligence (AI) technology within the field of graphic design currently include Adobe's Creative Cloud software, which is able to analyze the content of an image or video and make "intelligent recommendations" in order to automate "time-consuming" aspects of design [5]. And, looking ahead, Autodesk is presently developing Project DreamCatcher - a generative design system that will be capable of producing thousands of design options in a matter of seconds and "play an active, participatory role in the invention of form" [6]. 
Of course, these advancements have elicited polarizing views in the field of design. Typically, debates center around employability and economic productivity, with factions on one side arguing that by relegating time-consuming production activities to computers, designers will be able to expedite their creative work

[7]. Others, however, warn of a dystopian scenario with widespread unemployment resulting from increased automation [8].

However, outside of this binary rhetoric, and the pitting of the 'technophobic humanist' against the 'inhuman technologist' [9], what is less often discussed are the potential ramifications of $\mathrm{Al}$ technology in relation to the craft of graphic design practice.

Fig. 1. Michael Barnes Work (Source: https:// barnes.persona.co/)

Fig. 2. Michael Barnes Biography (Source: https://barnes.persona. $\mathrm{co} /)$

\section{ब캐을 VORTE}

INDEX

The Vanishing Future

Visual Identity

A 'The Vanishing Future' is a design festival in Seoul that needed a new logotype. It consists of a poetic visual identity that is both meaningful and classic. Main image is used as an advertising, a symbol to develop the functional ethos of festival.

\section{MIOHAEL} $=4 \mathrm{RN}=\mathrm{G}$
Within this rapidly-evolving and highly-contested environment, designer Sekyeong Kwon sought to encourage debate and make visible core concerns around $\mathrm{Al}$-powered design, from a graphic design perspective.

The resulting practice-led project, Michael Barnes, is outlined below.

\section{Michael Barnes}

Michael Barnes is a self-titled portfolio website (https:// barnes.persona.co/) which includes a manifesto, a curriculum vitae, and a gallery of design work featuring corporate identity, packaging and branding projects (See

MANIFESTO - UORK - UCV

凶 futureismichael@gmail.com

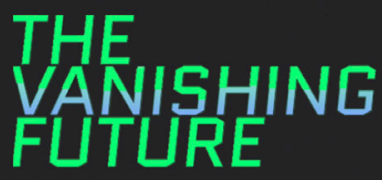

凶 futureismichael@gmail.com

๑ +44 (0)7380 262154

MANIFESTO - WORK - ÐCV
Michael Barnes is an award-winning design critic and visual communicator based in San Francisco and New York. His work places an experimental emphasis on installation, digital art, web design and typography. Ever since he moved to London, he started to seek finding ways to apply emerging

technologies to encourage our humanness, and ultimately to design a better world. He holds an MA in Communication Design from Falmouth university. 
Fig. 1). According to his biography, Barnes' is "an awardwinning design critic and visual communicator based in San Francisco and New York" (See Fig. 2).

Despite the unremarkable design work and the uncomfortable syntax, the website seems to all intents and purposes authentic. That is, the portfolio appears typical of the countless online design portfolios that are (arguably) largely indistinguishable from one another [10].

However, 'Michael Barnes', his name, biography, manifesto, and projects are entirely fictitious. ' $\mathrm{He}$ ' has been computationally generated using a combination of tools (Python coding, Markov Chain sequencing, image generators, etc.) and data scraped from existing graphic design texts and websites.

Thus, 'Barnes' represents but one randomized version of a potentially exponential number of designers and portfolios that could have been algorithmically generated. He could have, for example, been just as easily characterized as:

... a curious interaction designer and UI/UX designer based in Barcelona and Bangkok...

Or:

... a meticulous design strategist and visual communicator based in London and Sydney...

Certainly, this kind of parafictional deceit in cultural production is nothing new; Nat Tate (created by William Boyd) and The Yes Men (Jacques Servin and Igor Vamos) spring to mind. In each case, the characters draw on and mimic existing dialects - cultural, aesthetic, and textual - as a means of gaining entry to, and legitimacy within, a specific sphere [11]. However, unlike The Yes Men (et al) who intentionally 'dupe' the viewer, 'Michael Barnes' offers up 'his' own duplicity for scrutiny. Namely, the last section of the website, the curriculum vitae, reveals the project as a fictional endeavor, and makes publicly available the various code and tools used in the creation of the character and his portfolio.

\section{An Adversarial Approach}

While not overtly political, Kwon's project can be seen as a form of adversarial design - a type of critical making which seeks to provoke debate through the speculative modelling of possible scenarios and sociopolitical configurations [12]. Specifically, once 'Barnes' is revealed as an algorithmically generated figure, the existing relationship between $\mathrm{Al}$ technology and design, and perhaps more importantly, its shared future, are visibly problematized for the viewer. By doing so, rather than simply exploring the potential applications of Al technology and design, Michael Barnes demands consideration of its implications as well.

One area highlighted by Michael Barnes is the ease with which it achieves, at least in part, a kind of semiotic invisibility. It appears authentic. Thus, the website exposes the generic globalized reality of design and portfolio websites in which imitation has become a tool of legitimization. This, in turn, raises further questions around the current impact of Al technology in the field of design. Firstly, given that the featured work ('best of', 'most viewed' etc.) on graphic design showcase sites (for example, Behance and Dribbble), and the practice of locating stock images and templates, is search engine driven, to what degree are computers already agents of (rather than simply aids to) design practice? And looking forward, if design software is increasingly left to make decisions computationally, what will these be based on? Aggregates of taste? Engagement levels? Sponsorship? And, in turn, whose views will these privilege?

As such, the project recognizes that advancements in graphic design $\mathrm{Al}$ are not value-free, but rather, embedded within a broader data environment, which will ultimately prioritize particular social, political and economic forces.

Another area underscored by the project are shifting notions of originality within our current hypernetworked culture. Specifically, while Michael Barnes' portfolio is comprised entirely of pre-existing data, it is original in the sense that it is one-of-a-kind. In turn, this begs the question: who is the author of the website? The algorithm? Kwon? The 'original' authors of the now-unrecognizable data sources? And, additionally, who owns the intellectual property rights to the work? 
While theorization around shared cultural production and ownership recognizes contemporary appropriative cultural practices and the use of existing data as 'material' for production (such as sampling by deejays), the discourse is still predicated on human-agency and intentionality, which does little to clarify ongoing debates around $\mathrm{Al}$ and algorithmic authorship [13-14].

\section{Contestation vs Consensus}

Michael Barnes is by no means a 'polished' form of $\mathrm{Al}$; the design and various textual elements are largely inexpert; and the final outcome relied on the assembly of its individual parts by Kwon. However, the intention of the project is not to predict the future, nor to attempt to compete with rapid technological advancements in the field of computer science. Rather it seeks, playfully, to provoke debate and speculate 'what if?'. By suggesting a possible world, where a new 'designer' complete with personal backstory, ethos and portfolio can be generated at the click of a button, ad infinitum, the project encourages a recognition of the tensions which lie at the heart of the convergence of $\mathrm{Al}$ and graphic design.

In turn, Michael Barnes seeks a response from design educators and practitioners, a community whose voice is more often than not lost in the scientized race for Al. This provocation is, perhaps, most succinctly encapsulated in the darkly comic automated email response should you try to get in touch with 'him':

Hello! Thank you for your email.

BAD NEWS: I'm away from the office until Artificial General Intelligence becomes a reality.

GOOD NEWS: Me being away means that your job is safe from automation - for now.

Until then, Cheers!

Michael

\section{References}

1. Liebermann, H. (1996). Intelligent Graphics. Communications of the ACM, 39.

2. Ward, J. E., \& Ross, D. (1968). Computer-Aided Design for Numerically Controlled Production.

3. Nightmare Machine, http://nightmare.mit.edu/

4. Deep Dream Generator, https://deepdreamgenerator.com/

5. Adobe Sensei, https://www.adobe.com/sensei/creativecloud-artificial-intelligence.html

6. Project Dreamcatcher, https://autodeskresearch.com/ projects/dreamcatcher

7. Shaughnessy, A. (2017). Summoning the Demon? How Safe Are Creative Jobs from Automation? Creative Review.

8. Burgoyne, P. (2017). Al and creatives' complicity in their own extinction? Creative Review

9. Agüera y Arcas, B. (2017). Art in the Age of Machine Intelligence. Arts, 16(8).

10. Santos, M. (2016). The Unbearable Homogeneity of Design. Medium.

11. Sholette, G. (2011) Dark Matter: Art and Politics in the Age of Enterprise Culture. London: PlutoPress.

12. DiSalvo, C. (2012). Adversarial Design. Cambridge: The MIT Press.

13. Bourriaud, N. (2001) Postproduction. New York: Lukas \& Steinberg.

14. Kaminsky, M. (2017) Authorship, Disrupted: Al Authors in Copyright and First Amendment Law. UC Davis Law Review, 51(589). 